Simulations of Fracture and Fragmentation of Geologic Materials using Combined FEM/DEM Analysis

J. P. Morris, M. B. Rubin, G. I. Block, M. P. Bonner

May 31, 2005

Hypervelocity Impact Symposium 2005

Lake Tahoe, CA, United States

October 10, 2005 through October 14, 2005 
This document was prepared as an account of work sponsored by an agency of the United States Government. Neither the United States Government nor the University of California nor any of their employees, makes any warranty, express or implied, or assumes any legal liability or responsibility for the accuracy, completeness, or usefulness of any information, apparatus, product, or process disclosed, or represents that its use would not infringe privately owned rights. Reference herein to any specific commercial product, process, or service by trade name, trademark, manufacturer, or otherwise, does not necessarily constitute or imply its endorsement, recommendation, or favoring by the United States Government or the University of California. The views and opinions of authors expressed herein do not necessarily state or reflect those of the United States Government or the University of California, and shall not be used for advertising or product endorsement purposes. 


\title{
Simulations of Fracture and Fragmentation of Geologic Materials using Combined FEM/DEM Analysis
}

\author{
J. P. Morris ${ }^{\mathrm{a}}$, M. B. Rubin ${ }^{\mathrm{b}}$, G.I. Block ${ }^{\mathrm{a}}$, and M. P. Bonner ${ }^{\mathrm{a}}$ \\ ${ }^{a}$ Earth Sciences Division \\ Lawrence Livermore National Laboratory \\ Livermore, CA 94550 \\ morris50@1lnl.gov, block4@1lnl.gov, bonner10@1lnl.gov \\ ${ }^{b}$ Faculty of Mechanical Engineering, \\ Technion - Israel Institute of Technology \\ 32000 Haifa, Israel \\ mbrubin@tx.technion.ac.il
}




\begin{abstract}
Results are presented from a study investigating the effect of explosive and impact loading on geological media using the Livermore Distinct Element Code (LDEC). LDEC was initially developed to simulate tunnels and other structures in jointed rock masses with large numbers of intact polyhedral blocks. However, underground structures in jointed rock subjected to explosive loading can fail due to both rock motion along preexisting interfaces and fracture of the intact rock mass itself. Many geophysical applications, such as projectile penetration into rock, concrete targets, and boulder fields, require a combination of continuum and discrete methods in order to predict the formation and interaction of the fragments produced. In an effort to model these types of problems, we have implemented Cosserat point theory and cohesive element formulations into the current version of LDEC, thereby allowing for dynamic fracture and combined finite element/discrete element simulations. Results of a large-scale LLNL simulation of an explosive shock wave impacting an elaborate underground facility are also discussed. It is confirmed that persistent joints lead to an underestimation of the impact energy needed to fill the tunnel systems with rubble. Non-persistent joint patterns, which are typical of real geologies, inhibit shear within the surrounding rock mass and significantly increase the load required to collapse a tunnel.
\end{abstract}

\title{
1. Introduction
}

A wide range of geological applications involve materials or systems that are discontinuous at a small enough scale of observation. While some systems may be intrinsically discontinuous, other discontinuous systems are best approximated by a continuum, a discontinuum, or a combination of the two, depending upon the specific information of interest. Continuum, mesh-based methods have been applied successfully to many problems in geophysics, and a continuum approximation may be adequate when sufficiently large length scales are considered - even if the geology includes fractures and faults. However, a large class of problems exists where individual rock discontinuities must be taken into account. This includes problems whose structures of interest have sizes 
comparable with the block size, or when the structures experience loads that do no measurable damage to individual blocks, but deformation along material discontinuities still leads to structural failure. In these cases, a purely continuum, mesh-based treatment is usually inappropriate. Field tests indicate that structural response can be dominated by the effect of preexisting fractures and faults in the rock mass, as shown in Figure 1. Consequently, accurate models of underground structures must take into account deformation across fractures and not simply within the intact portions of the rock mass.

The distinct element method (DEM) is naturally suited to simulating such systems because it can explicitly accommodate the blocky nature of natural rock masses. Cundall and Hart (1992) review a number of numerical techniques that have been developed to simulate the behavior of discontinuous systems using DEMs. In general, an explicit scheme is used to evolve the equations of motion of discrete bodies directly and the bodies may be rigid or deformable. Early approaches employed rigid disks or spheres with compliant contacts (Cundall and Strack, 1979; Cleary, 1991). Cundall, (1980) Walton, (1980) and Cundall and Hart (1985) developed two-dimensional DEMs that employed arbitrary polygons. Cundall (1988) and Hart et al. (1988) also developed fully three-dimensional DEMs composed of rigid or deformable polyhedral blocks with compliant contacts.

The DEM has been applied to a wide range of problems in geomechanics [see Cundall (2001) for a review of DEM simulations of granular material and rock]. In particular, Antonellini and Pollard (1995) simulated the formation of shear bands in sandstone using the DEM. Morgan (1999a, 1999b) applied the DEM to the mechanics of granular shear zones, and Heuze et al. (1993) used the DEM method to analyze explosions in hard rock. Sanderson and Zhang (1998) also used a two-dimensional distinct element method to investigate the evolution of damage zones and fluid flow in fracture networks and around faults.

One fundamental advantage of the DEM is that pre-existing joints in rock can be incorporated into a DEM model directly, and the joints are allowed to undergo large deformation. Detailed joint constitutive models [see Morris (2003) for a review] can also 
be used to combine experimentally observed fracture properties (such as joint dilation, friction angle, and cohesion) with the DEM approach. However, many applications in geophysics require a combined continuum-discontinuum treatment for a complete solution. For example, projectile penetration into a rock or concrete target requires continuum-discontinuum analysis in order to predict the formation and interaction of the fragments produced. The impact of a penetrating weapon into a boulder field also lends itself to solution by a combination of continuum and discrete methods. Underground structures in jointed rock subjected to explosive loading can fail due to both rock motion along preexisting interfaces and fracture of the intact rock mass itself. In such applications, it is insufficient to simply predict whether or not the rock mass with failinstead, the critical issues are how fracture and discontinuous interaction lead to the ultimate fate of rock fragments.

To answer these questions, a continuum-discontinuum capability was developed by incorporating finite element analysis and cohesive elements into the Livermore Distinct Element Code (LDEC). This computer code was originally developed by Morris et al. (2002) to simulate the response of jointed geologic media to dynamic loading. Section 2 presents an outline of the Cosserat point theory that underlies the current version of LDEC. A discussion is also presented of preliminary work on a cohesive element formulation that will allow the study of fracture problems in the continuum-discontinuum setting. Sections 3 and 4 focus on results from simulations of fracture and fragmentation for the case of a shock wave impacting an underground tunnel system. In conclusion, Section 5 presents a short discussion of the influence of persistent and non-persistent joint sets on failure in rock and the versatility of combined continuum-discontinuum approaches.

\section{Treatment of Jointed Media using LDEC}

In the simplest case, the Livermore Distinct Element Code can be run in rigid-block mode, so that all deformation in the system is lumped into the contacts. The most complicated aspect of the code is then related to contact detection. In general, the 
equations of motion of the elements are determined in a standard manner by integrating vector equations for both the center of mass of each element and an orthonormal vector triad that determines its absolute orientation. Contact detection monitors how the connectivity changes as a result of relative block motion. The Lagrangian nature of the DEM also simplifies tracking of material properties as blocks move, and it is possible to guarantee exact conservation of linear and angular momentum throughout the computation.

\subsection{Cosserat Point Formulation}

Deformation within the individual blocks is often introduced into DEM formulations by using additional standard continuum discretization. For example, Cundall (1980, 1988) and Hart et al. (1988) use finite differences to model the deformation of compliant blocks. In Morris et al., (2004) it was observed that the theory of a Cosserat point (Rubin, 1995, 2000) can model each element as a homogenously deformable continuum. A Cosserat point describes the dynamic response of the polyhedral rock block by enforcing a balance of linear momentum to determine the motion of the center of mass (3 translational degrees of freedom), as well as three vector balance laws of director momentum to determine a triad of deformable vectors, which model both the orientation of the element (3 rotational degrees of freedom) and its deformation (6 degrees of freedom for dilatation and distortion). The response of the deformable polyhedral block is modeled explicitly using the standard material constants that characterize the original three-dimensional material, and constitutive equations for the contact forces at the joints become pure measures of the mechanics of joints.

The version of LDEC that includes homogeneously deformable Cosserat points has been used successfully to model a number of problems of physical interest (Morris et al., 2004). However, this approach is inappropriate for problems whose length scales of interest (such as a tunnel diameter) are only slightly greater than the block size. This deficiency was overcome by internally discretizing the polyhedral blocks with a collection of smaller tetrahedral elements. The numerical solution procedure depends on 
nodal balance laws to determine the motion of the four nodes of each tetrahedral element, similar to that described above for the motion of blocks. In general, the accelerations of the nodes of a particular element are coupled with the nodes of the neighboring elements. However, the director inertia coefficients in the theory of a Cosserat point can be specified so that these equations become uncoupled. This form corresponds to a lumped mass assumption and is particularly convenient for wave propagation problems using explicit integration schemes because it does not require the inversion of a stiffness matrix.

In continuum regions, where the nodes of neighboring elements are forced to remain common (i.e., unbreakable), the Cosserat point formulation is basically the same as standard finite element models (FEM) that use homogeneously deformable tetrahedral elements. In this case, the computational effort in LDEC is significantly reduced: many nodes are shared and there is no need for contact detection on shared element surfaces. While standard finite element formulations are based on shape functions and weighting functions, the latest version of LDEC utilizes balance laws for the directors of each Cosserat point (associated with the positions of the nodes of the tetrahedral elements). LDEC can be run simultaneously in DEM and FEM-like modes, dynamically blending continuum and discrete regions, as necessary.

\subsection{LDEC Fracture Algorithm}

The research described here focuses on extending the advantages of both continuum and discontinuum descriptions to include dynamic fracture in jointed and heterogeneous rock. First, a volume-weighted average Cauchy stress is calculated in the elements surrounding each node. At each time step, LDEC determines the principal stresses $\sigma_{i}$ (positive indicates tension) and the associated principal directions $\mathbf{p}_{i}$, ordered such that

$$
\sigma_{1} \geq \sigma_{2} \geq \sigma_{3} .
$$

When $\sigma_{1}$ first equals the failure stress $T_{f}$ of the medium, the node is split into two daughter nodes that are connected by a special "cohesive" element, as shown in Figure 
2(a). Figure 2(b) depicts the weakening force law that is used to simulate the process of nodal de-cohesion (Dugdale, 1960; Barrenblatt, 1962). This approach is somewhat different from that taken by $\mathrm{Xu}$ and Needleman (1994) and Camacho and Ortiz (1996), for example, who focus on the separation of surfaces instead of nodes and who include combined effects of failure in tension and/or shear.

The remaining nodes that are directly connected to the daughter nodes by edges of the neighboring elements can be tested to determine if they become new exterior nodes or remain interior nodes based on the criterion that a newly created fracture surface will have its normal closest to the principal direction associated with the failure. In this way, the fracture surface will open up along those existing element boundaries that are most closely aligned with the plane of largest (tensile) principal stress.

In order to compute the evolution of each node, consider $\mathbf{x}_{K}$ to be the virtual position of the previously "unbroken" node $K$, and let $\mathbf{x}_{A}$ and $\mathbf{x}_{B}$ be the positions of the daughter nodes $A$ and $B$. Each of these nodes is surrounded by a part consisting of neighboring elements. Now, define $\mathbf{f}_{B / A}$ to be the force applied by the part associated with $A$ on the part associated with $B$, and define the converse, $\mathbf{f}_{A / B}$, similarly. Also, let $\mathbf{F}_{A}$ be the force applied to $A$ due to the elements connected to $A$ and let $\mathbf{F}_{B}$ be the force applied to $B$ due to the elements connected to $B$. If the effective mass of nodes $A$ and $B$ are $m_{A}$ and $m_{B}$, respectively, then the equations of motion for these nodes are simply:

$$
\begin{aligned}
& m_{A} \frac{d^{2} \mathbf{x}_{A}}{d t^{2}}=\mathbf{F}_{A}+\mathbf{f}_{A / B} \\
& m_{B} \frac{d^{2} \mathbf{x}_{B}}{d t^{2}}=\mathbf{F}_{B}+\mathbf{f}_{B / A},
\end{aligned}
$$

where $\mathbf{f}_{B / A}=-\mathbf{f}_{A / B}$. Before the initiation of fracture, the motion of node $K$ is given by adding the equations in (2) together, subject to the constraint that $\mathbf{x}_{A}=\mathbf{x}_{B}=\mathbf{x}_{K}$. Thus,

$$
\frac{d^{2} \mathbf{x}_{K}}{d t^{2}}=\frac{\mathbf{F}_{A}+\mathbf{F}_{B}}{m_{A}+m_{B}},
$$

and the cohesive force $\mathbf{f}_{A / B}$ is given by 


$$
\left.\mathbf{f}_{c} \equiv \mathbf{f}_{A / B}\right|_{\text {fracture }}=\frac{m_{A} \mathbf{F}_{B}-m_{B} \mathbf{F}_{A}}{m_{A}+m_{B}},
$$

when the daughter nodes are first created.

In this work, de-cohesion is considered as a central-force process derived from the weakening law depicted in Figure 2(b). Let $d(t)$ be the distance between the points $\mathbf{x}_{A}$ and $\mathbf{x}_{B}$, so that

$$
d=\left\|\mathbf{x}_{B}-\mathbf{x}_{A}\right\| .
$$

The cohesive force directed between the daughter nodes, $\mathbf{f}_{A / B}=f \mathbf{e}_{B / A}$, depends on the unit vector $\mathbf{e}_{B / A}=\frac{1}{d}\left(\mathbf{x}_{B}-\mathbf{x}_{A}\right)$ and the weakening law:

$$
f=\alpha f_{c}\left(1-\frac{\delta_{\max }}{\delta_{c}}\right) \frac{d}{\delta_{\max }} .
$$

Here, $f_{c}=\left\|\mathbf{f}_{c}\right\|$ is calculated at the onset of fracture using (4) and remains constant. The coefficient $\alpha$ determines whether the forces that cause daughter nodes to separate are continuous in time $(\alpha=1)$ or are discontinuous in time $(0 \leq \alpha<1)$ [see Papoulia et al. (2003)]. Finally,

$$
\delta_{\max }=\min \left(d_{\max }, \delta_{c}\right)
$$

and

$$
d_{\max }=\max \left(d, d_{\max }\right)
$$

are calculated at each time step to allow for reversible unloading and reloading along a linear path from the origin to the most recent position on the (weakening) forcedisplacement curve. The singularity arising in (6) when $\delta_{\max }$ vanishes is removed by simply setting $d / \delta_{\max }=1$ in that case.

\subsection{Effective Properties in Crack Propagation}

Cohesive elements characterize the averaged deformation in a region around the crack tip. Integrating the power dissipated by the cohesive element from initiation to failure 
(whence $\delta_{\max }=\delta_{c}$ ) leads to an expression for the total work done by the element, which is denoted by $W_{c}$. Let $A_{f}$ be the total surface area associated with the newly created fracture surface (including both faces of the new fracture surface) and let $\Gamma$ be the fracture energy per unit area associated with creating this surface. Therefore,

$$
-W_{c}=\frac{1}{2} \alpha f_{c} \delta_{c}=A_{f} \Gamma,
$$

so that the critical opening displacement $\delta_{c}$ of the cohesive element is given by

$$
\delta_{c}=\frac{2 A_{f} \Gamma}{\alpha f_{c}} .
$$

Length scales analogous to $\delta_{c}$ serve as effective measures of the size of a cohesive zone around the crack-tip singularity, where linear elasticity breaks down (Freund, 1990).

Averaging over this zone can remove the singularity, but it necessarily introduces additional models of plastic flow and inelasticity (such as the weakening law). Typical values of the critical displacement can be deduced from experiments using a continuum version of (10), and are found to be approximately $10 \mu \mathrm{m}$ in PMMA and $10 \mathrm{~cm}$ in concrete (Ruiz et al., 2000). Many FEM fracture computations utilize a mesh resolution that is significantly finer than the smallest length scale in the simulation, $\delta_{c}$, because field variations on the scale of the cohesive zone can lead to dynamical instabilities and branching, which are not always observable on the macroscale. Indeed, some branching processes are initiated at critical crack-tip velocities that may be universal features of brittle fracture in isotropic, homogeneous media (Sharon and Fineberg, 1996).

LDEC runs for large-scale geophysical problems will be unable to resolve the dynamics near the crack tip. Instead, simulations rely on straight, flat cracks, as depicted in Figure 3. This trajectory represents a loss of microscale information: the true path length and total exposed area, as well as the effective fracture energy, $\bar{\Gamma}(\overline{\mathbf{v}})$, depend on both the scale and the effective crack-tip velocity $\overline{\mathbf{v}}$. Preliminary results for brittle fracture in PMMA plates are shown in Figures 4(a) and 4(b), where an applied stress is used to initiate failure using a seed crack at the plate's center. Cohesive elements are 
constrained to lie along the middle line only, and a constant value of $\Gamma$ was used (the mesh resolution is $1000 \delta_{c}$ ). The crack-tip velocity quickly asymptotes to a value near the Rayleigh wavespeed, just as expected for straight cracks (Fineberg and Marder, 1998). Crack branching is observed when the initiation of cohesive elements is unconstrained. Current research is focusing on comparing these behaviors to experimental data and developing techniques to use observed values of $\bar{\Gamma}(\overline{\mathbf{v}})$ to remove the mesh dependence that arises as an artifact of spatial averaging.

\section{Simulations of an Underground Facility in Fractured Rock}

A series of simulations were recently performed on the "Thunder" supercomputer at Lawrence Livermore National Laboratory to model the effects of a surface explosion (and subsequent shock wave) on an underground tunnel system surrounded by jointed rock. In practice, the extent of an LDEC simulation is limited by the computational effort required to simulate the necessary number of rock blocks. Thunder provides a maximum of 4008 Itanium processors, allowing us to consider models of greater size and complexity than had previously been possible.

The solution domain spanned $60 \mathrm{~m}$ in each direction and encapsulated a generic facility that included several tunnel sections and a lift shaft (see Figure 5). Several geological models were considered as part of this study. In particular, the behavior of regular, persistent joints was compared to the effect of non-persistent (i.e., randomized) joints in the surrounding rock. The rigid block capability was used to model hard rock and to emphasize the role of joint geometries, but cohesive elements were not included in these simulations. Figure 6 shows an example of the randomized jointing present in the non-persistent model geology. In both cases discussed here, the joint patterns resulted in typical block sizes of $30 \mathrm{~cm}$. Consequently, each model contained approximately 8 million individual polyhedral rock blocks and approximately 100 million contact elements, making these the largest simulations of this type performed to date. The 
facilities were subjected to loading corresponding to one kiloton at the surface $50 \mathrm{~m}$ above.

Figure 7 compares the velocity fields of the two simulations at $30 \mathrm{~ms}$. Results obtained for the regular, persistent joint set and irregular, non-persistent model differed in several key ways:

- The regular model exhibited strong anisotropy. Since the joints are weak under shear loading, the regular, persistent joint sets tend to channel the waveform, resulting in variations in wavespeed with direction of propagation.

- The irregular model exhibited higher attenuation. Again, because the joints are weak under shear loading, the irregular joint structure results in more effective plastic deformation on the joints and, consequently, more attenuation.

- Persistent joints allow shear motion along the entire length of the computational domain, resulting in large "chimney" effects above collapsed tunnels sections.

- The irregular model resulted in more diffraction of waves around cavities in the rock mass.

Figure 8 shows two snapshots of the collapse of the largest room within the facility using the non-persistent joint set simulation. While the largest room within the facility has totally collapsed, the narrowest access tunnels experienced minimal damage. The midsize tunnels show a range of damage, with most damage occurring in tunnel sections that contain a junction with another tunnel or lift shaft. This behavior is consistent with the idea that tunnel junctions compromise tunnel strength.

\section{Simulations of a Tunnel Opening in Infrequently Jointed Rock}

Previous LDEC runs have modeled tunnels in heavily jointed, hard rock, where the tunnel diameter was spanned by many blocks. It is then appropriate to simulate the rock mass using a "tight" structure consisting of polyhedral blocks that are either rigid or homogenously deformable (with deformable points of contact in both cases). In contrast, the focus of this section is on a class of problems where the joints are sufficiently 
infrequent so that the predominant failure mechanism is block breakage rather than intact rock displacement.

This preliminary simulation is performed in two dimensions. The geology consists of blocks of limestone, measuring $1.83 \mathrm{~m}$ wide, by $0.30 \mathrm{~m}$ high, surrounding a tunnel measuring $2.73 \mathrm{~m}$ by $2.80 \mathrm{~m}$. The tunnel is subjected to loading that corresponds to a tamped, one-ton detonation located $4.88 \mathrm{~m}$ left and $5.75 \mathrm{~m}$ above the tunnel. The calculation was performed in two stages. Initially, LDEC was run with deformable blocks of limestone internally discretized into $10 \mathrm{~cm}$ tetrahedral elements. Cohesive elements were not included. In this mode of operation, the time step is quite short so that modes of deformation within the $10 \mathrm{~cm}$ elements can be captured. After $10 \mathrm{~ms}$, the LDEC calculation was switched over to rigid-block mode, which ignores internal modes of the elements to increase the size of the time steps and to investigate the flow of rubble into the tunnel over long time scales. The simulation shows that a significant portion of the rock mass surrounding the tunnel is reduced to rubble by the tension produced as waves reflect from the interior surface of the tunnel. This rubble mass is sufficient to fill in the original tunnel.

\section{Conclusions}

The Livermore Distinct Element Code is capable of simulating the dynamic response of elaborate, underground facilities and tunnel systems to shock-wave loading. Such largescale studies allow the investigation of the interaction between different parts of the facility, and the study of how these interactions lead to tunnel collapse and overall failure. The results highlight the importance of including realistic irregular, non-persistent joint sets. Geologies that were modeled using rigid blocks with deformable contacts and nonpersistent jointing were found to withstand significantly more shear deformation than

would be predicted using regular joint sets. Moreover, discretizing the blocks internally with tetrahedral elements increases the potential accuracy of large-deformation simulations, especially in cases where the predominant failure mechanism is block breakage rather than intact rock displacement. 
Capturing the physics of fracture in heterogeneous media requires more than renormalization arguments based on lattice approximations (Nguyen and Ortiz, 2002). Also, scaling laws will be critical for removing mesh dependence in the simulations. The current version of LDEC provides simultaneous DEM and FEM-like domain partitioning, as well as the possibility of converting between the two modes dynamically. Future work will focus on combining the DEM, FEM, and cohesive elements together to produce a efficient formulation that is both accurate and robust. Specifically, it is anticipated that this effort will produce the capability of large-scale simulations of fragmentation and dynamic fracture of an important class of problems that are simply beyond the scope of current codes.

\section{Acknowledgments}

This research was performed under the auspices of the U.S. Department of Energy by Lawrence Livermore National Laboratory under contract No. W-7405-Eng-48. M.B. Rubin was partially supported by the Fund for Promotion of Research at Technion. Also, the authors would like to acknowledge helpful discussions with L. Glenn and T. Antoun.

This work was performed under the auspices of the U.S. Department of Energy by University of California, Lawrence Livermore National Laboratory under contract W-7405-Eng-48. 


\section{References}

Antonellini, M. A. and Pollard, D. D. (1995), Distinct element modeling of deformation bands in sandstone, J. Struct. Geol., 17, 1165-1182.

Barrenblatt, G. I. (1962), The mathematical theory of equilibrium of cracks in brittle fracture, $A d v$. Appl. Mech., 7, 55-129.

Camacho, G. T. and Ortiz, M. (1996), Computational modeling of impact damage in brittle materials, Int. J. Solids Structures, 33, 2899-2938.

Cleary, P. W. (1991), Extensions of the hybrid method for granular flows, Proceedings 5th International Computational Techniques and Applications Conference, Adelaide, Australia.

Cundall, P. A. (1980), UDEC-A generalized distinct element program for modeling jointed rock, Final Tech. Rep. Eur. Res. Office (US Army Contract DAJA37-79-C-0548), NTIS order No. AD-A087 $610 / 2$.

Cundall, P. A. (1988), Formulation of a three-dimensional distinct element model - Part I. A scheme to detect and represent contacts in a system composed of many polyhedral blocks, Int. J. Rock Mech. Min. Sci. Geomech. Abstr., 25, 107-116.

Cundall, P. A. (2001), A discontinuous future for numerical modeling in geomechanics?, Proc. Inst. Civ. Eng-Geotech. Eng., 149, 41-47.

Cundall, P. A. and Hart, D. H. (1992), Numerical modeling of discontinua, Eng. Comput., 9, 101-113.

Cundall, P. A. and Hart, R. D. (1985), Development of generalized 2D and 3D distinct element programs for modeling jointed rock, Misc. Paper SL-85-1, US Army Corps of Engineers, SL-85-1.

Cundall, P. A. and Strack, O. D. L. (1979), A discrete numerical model for granular assemblies, G'eotechnique, 29, 47-65.

Dugdale, D. S. (1960), Yielding of steel sheets containing slits, J. Mech. Phys. Solids, 8, 100-104.

Fineberg, J. and Marder, M. (1998), Instability in Dynamic Fracture, preprint submitted to Elsevier Preprint.

Freund, L. B. (1990), Dynamic Fracture Mechanics, Cambridge University Press, New York, NY.

Hart, R., Cundall, P. A., and Lemos, J. (1988), Formulation of a three-dimesional distinct element model Part II. Mechanical calculations for motion and interaction of a system composed of many polyhedral blocks, Int. J. Rock Mech. Min. Sci. Geomech. Abstr., 25, 117-125.

Heuze, F. E., Walton, O. R., Maddix, D. M., Shaffer, R. J., and Butkovich, T. R. (1993), Analysis of explosions in hard rocks: the power of discrete element modeling, in Comprehensive Rock Engineering, Hudson, J. A., Brown, E. T., Fairhurst, C., and Hoek, E., Pergamon Press, New York, NY, pp. 387-413.

Morgan, J. K. (1999a), Numerical simulations of granular shear zones using the distinct element method 1. 
Shear zones kinematics and the micromechanics of localization, J. Geophys. Res., 104, 2703-2719.

Morgan, J. K. (1999b), Numerical simulations of granular shear zones using the distinct element method 2. Effects of particle size distribution and interparticle friction on mechanical behavior, J. Geophys. Res., 104, 2721-2732.

Morris, J. P. (2003), Review of Rock Joint Models, Lawrence Livermore National Laboratory, UCRL-ID153650, http://www-r.llnl.gov/tid/lof/documents/pdf/244645.pdf.

Morris, J. P., Glenn, L. A., and Blair, S. C. (2002), The distinct element method - application to structures in jointed rock, in Lecture Notes in Computational Science and Engineering: Meshfree Methods, 26, Springer-Verlag, Heidelberg, pp. 291-306.

Morris, J. P., Rubin, M. B., Blair, S. C., Glenn, L. A., and Heuze, F. E. (2004), Simulations of underground structures subjected to dynamic loading using the distinct element method, Engng Comput., 21, 384408.

Nguyen, O. and Ortiz, M. (2002), Coarse-graining and renormalization of atomistic binding relations and universal macroscopic cohesive behavior, J. Mech. Phys. Solids, 50, 1727-1741.

Papoulia, K. D., Sam, C.-H., Vavasis, S. (2003), Time continuity in cohesive finite element modeling, Int. J. Numer. Meth. Engng, 58, 679-701.

Rubin, M. B. (1995), Numerical solution of two- and three-dimensional thermomechanical problems using the theory of a Cosserat point, in J. of Math. and Phys. (ZAMP), 46, Casey, J. and Crochet, M. J., Brikhauser Verlag, Basel, pp. S308-S334.

Rubin, M. B. (2000), Cosserat Theories: Shells, Rods and Points, Solid Mechanics and its Applications, Kluwer, The Netherlands.

Ruiz, G., Ortiz, M., and Pandolfi, A. (2000), Three-dimensional finite-element simulation of the dynamic Brazilian tests on concrete cylinders, Int. J. Numer. Meth. Engng, 48, 963-994.

Sanderson, D. J. and Zhang, X. (1998), Deformation, damage, and fluid flow in fracture networks and around faults, Fall Meeting of the American Geophysical Union,

Sharon, E. and Fineberg, J. (1996), Microbranching instability and the dynamic fracture of brittle materials, Phys. Rev. B, 54, 7128-7139.

Walton, O. R. (1980), Particle dynamics modeling of geological materials, Lawrence Livermore National Laboratory, UCRL-52915.

Xu, X.-P. and Needleman, A. (1994), Numerical simulations of fast crack growth in brittle solids, J. Mech. Phys. Solids, 42, 1397-1434. 


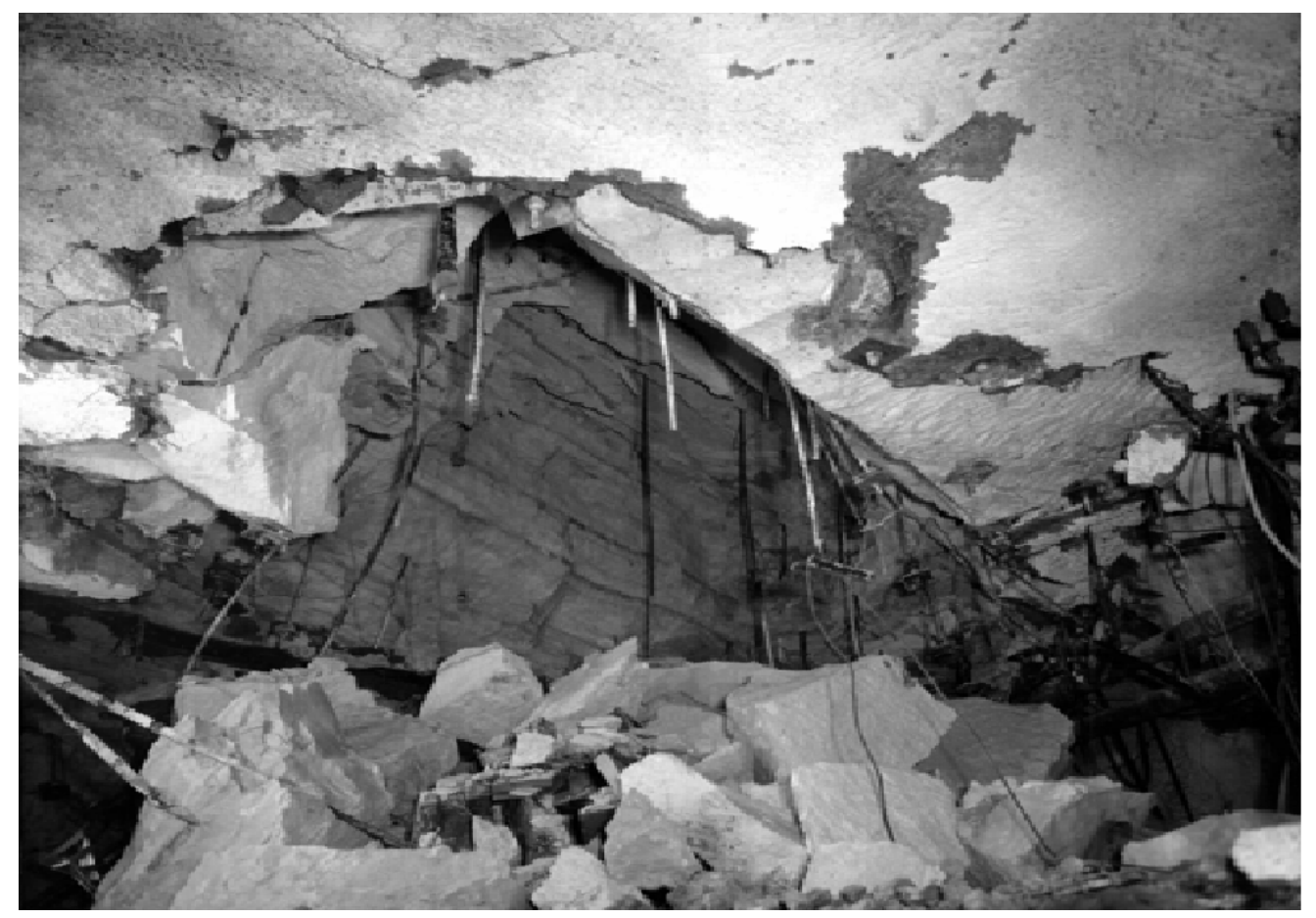

Figure 1: The blocky nature of the rock mass is evident in the collapse of this cavern in Tuff. This cavern collapsed at stresses below the yield strength of the intact rock mass.

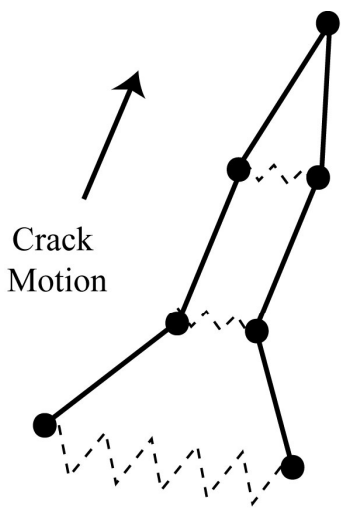

(a)

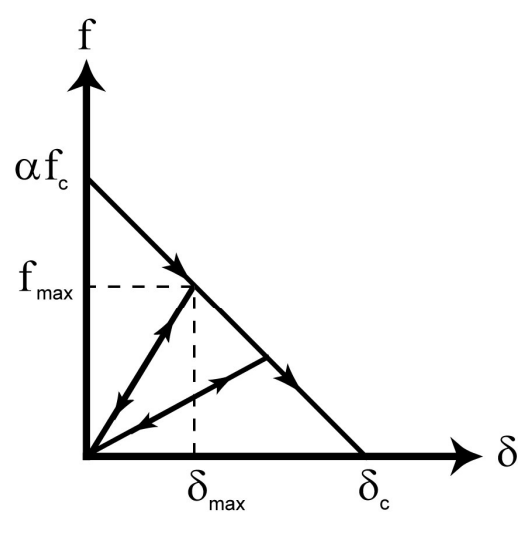

(b)

Figure 2: Cohesive elements. (a) Daughter nodes created near a crack tip; (b) weakening law, including reversible unloading and reloading paths to the origin. 


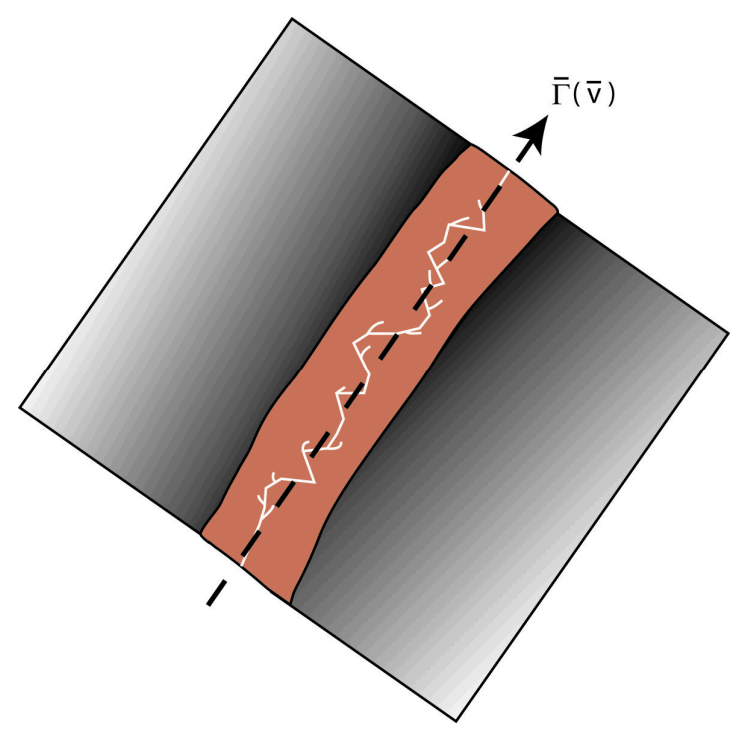

Figure 3: Scale- and velocity-dependent properties (such as the effective fracture energy, $\bar{\Gamma})$ arise naturally in large-scale simulations of crack propagation, which are often unable to resolve surface roughness and unstable branching on the microscale.

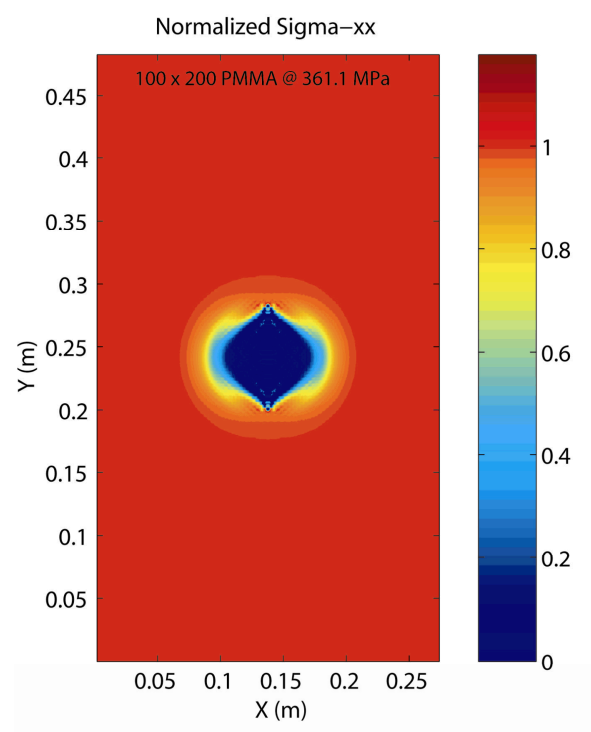

(a)

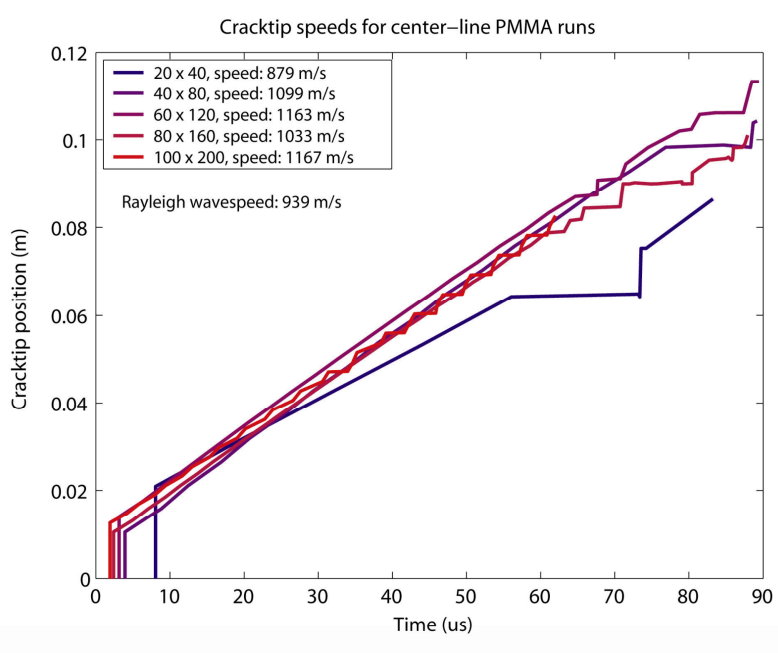

(b)

Figure 4: Center-line fracture in PMMA plates. (a) Applied tensile stress along the $\mathrm{x}$-axis causes a seeded crack to tear; (b) the resulting crack-tip speeds are close to the Rayleigh wavespeed, just as expected for straight cracks that cannot branch. 


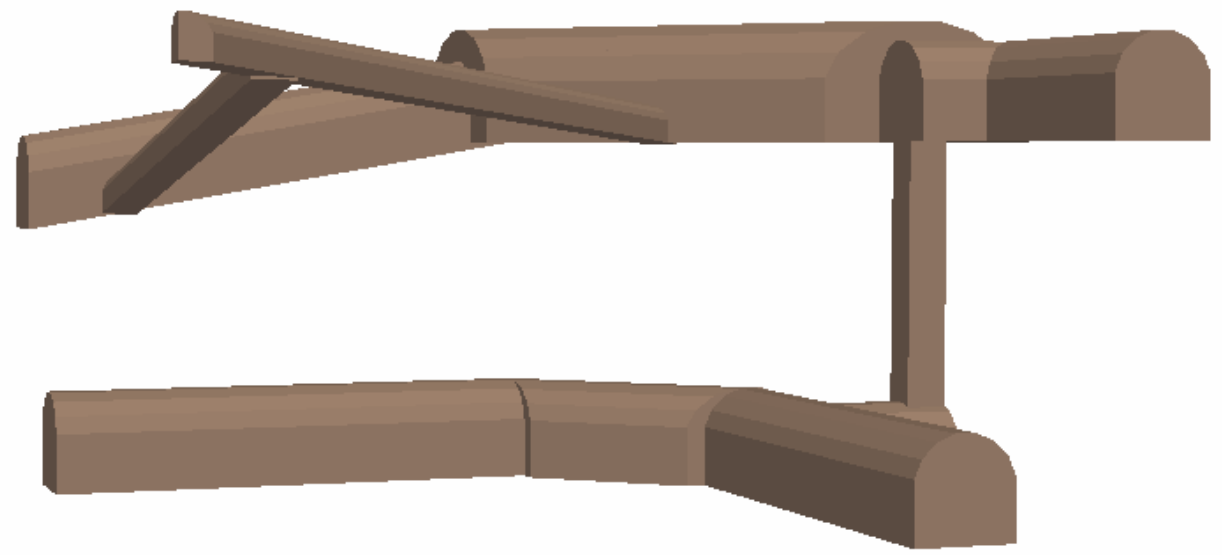

Figure 5: Generic facility model including several tunnel sections and a lift shaft. The facility spans $60 \mathrm{~m}$ and is $50 \mathrm{~m}$ below the surface.

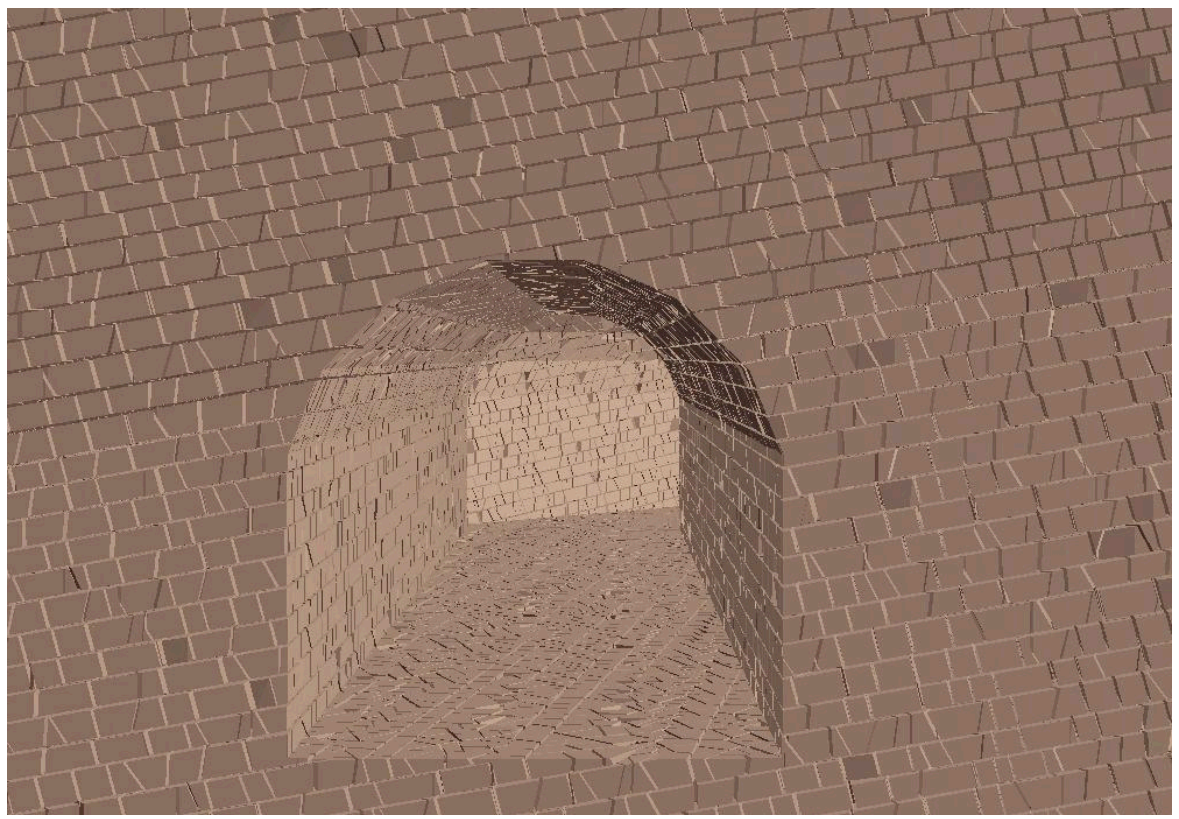

Figure 6: The non-persistent randomized geology in the vicinity of one of the tunnels. The near-horizontal joint set persists through the model. However, joint sets in the near vertical direction persist only through several consecutive layers at a time. 
$10 \mathrm{~m} / \mathrm{s}$
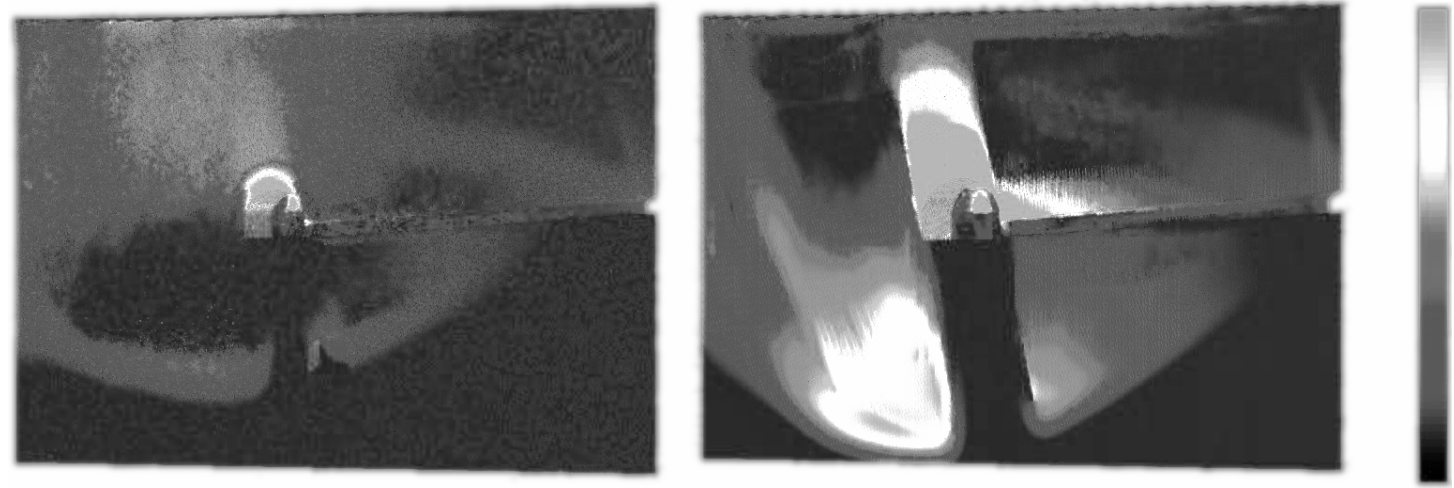

$0 \mathrm{~m} / \mathrm{s}$

Figure 7: The velocity magnitude for the two models at 30ms. The non-persistent, randomized geology model (left) and regular jointed model (right) exhibit fundamentally different responses to loading.
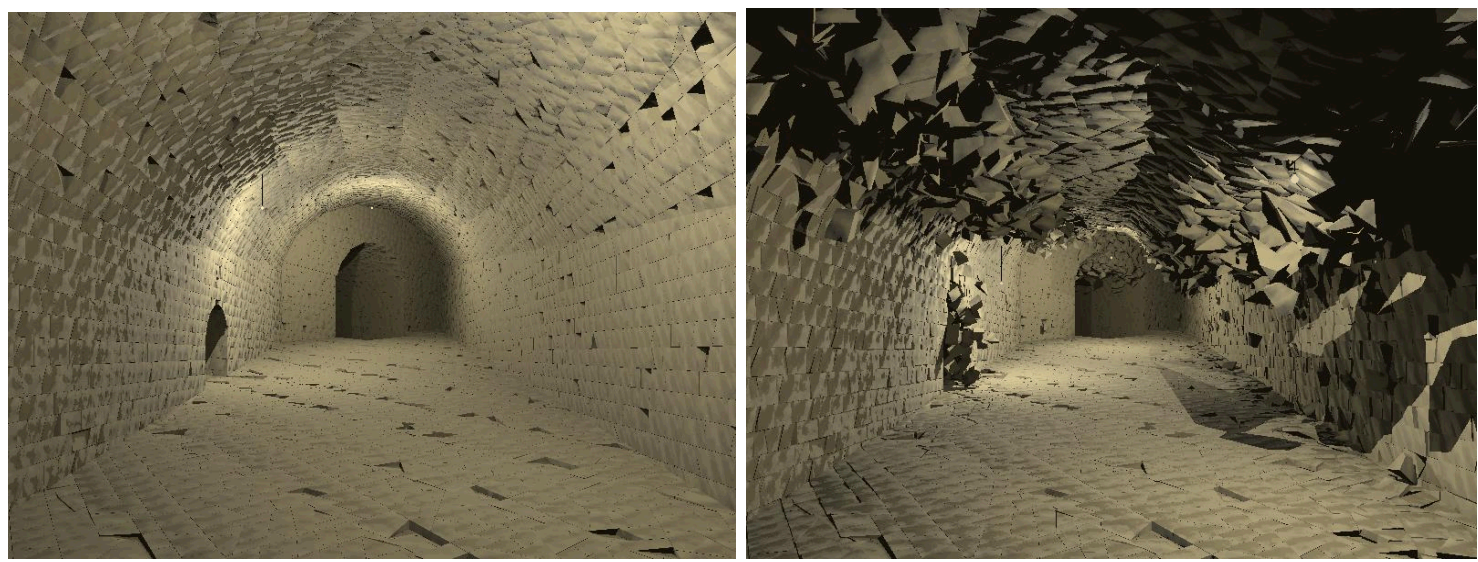

Figure 8: Snapshots of the largest room at $0 \mathrm{~ms}$ and $200 \mathrm{~ms}$, using the non-persistent joint set simulation. The simulation predicts that this large room within the facility would completely collapse under the applied loading. 

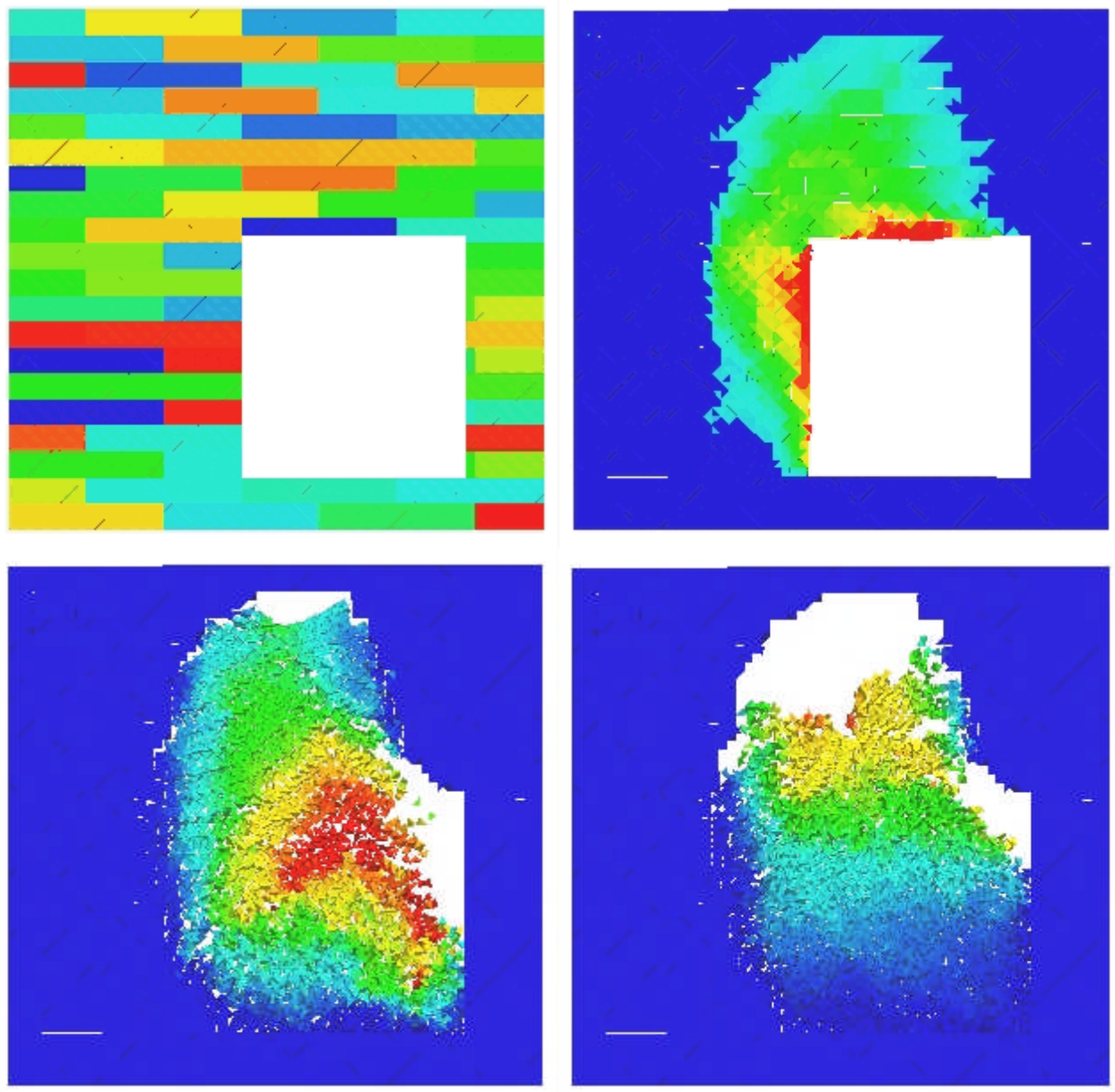

Figure 9: The infrequently jointed model is displayed top left, with individual blocks colored randomly to emphasize joint locations. The simulation results at $10 \mathrm{~ms}, 500 \mathrm{~ms}$ and $1000 \mathrm{~ms}$, show that a significant portion of the surrounding rock is reduced to rubble and fills the tunnel. Colors in the figures denote velocity magnitudes, with blue indicating $0 \mathrm{~m} / \mathrm{s}$ and red indicating $10 \mathrm{~m} / \mathrm{s}$. 